\title{
Respostas cardiorrespiratórias durante dois testes de exercício submáximos em participantes de um programa de reabilitação cardíaca: resultados preliminares
}

\author{
Marília Severo Vicente ${ }^{1}$ Tamires Daros dos Santos ${ }^{1}$ Viviane Acunha Barbosa ${ }^{2}$ Tiago José Nardi Gomes ${ }^{3}$ Sérgio Nunes
} Pereira ${ }^{4}$ Dannuey Machado Cardos $0^{5}$ Carine Cristina Callegaro ${ }^{6}$ Isabella Martins de Albuquerque ${ }^{7}$

\begin{abstract}
RESUMO
Introdução - A capacidade submáxima de exercício avalia a capacidade funcional do indivíduo, podendo ser mensurada pelo Teste de Caminhada de Seis Minutos (TC6m) e pelo Teste do Degrau de Quatro Minutos (TD4). Objetivo - Comparar as respostas cardiorrespiratórias e percepção de esforço entre o TC6m e o TD4 em pacientes submetidos à cirurgia de revascularização do miocárdio (CRM) participantes de um programa de reabilitação cardíaca. Metodologia - Estudo transversal composto por sete homens (58,71 $\pm 9,37$ anos). Foram analisadas as respostas cardiorrespiratórias (saturação periférica de oxigênio - $\mathrm{SpO} 2$; frequência cardíaca - FC; pressão arterial sistólica - PAS; pressão arterial diastólica - PAD), sensação de dispneia (SD) e sensação de fadiga de membros inferiores (SFMMII) por meio da Escala de Borg modificada. Os testes seguiram as diretrizes da American Thoracic Society (ATS 2002),). Resultados - Constatou-se diferença significativa $(p<0,05)$ nos deltas da PAS e PAD entre os testes. Conclusão - Nossos resultados sugerem que tanto o TC6m quanto o TD4 estimam a capacidade submáxima de exercício, porém o TD4 exige uma maior demanda metabólica, observada por maior incremento da pressão arterial.
\end{abstract}

Descritores: Doenças Cardiovasculares; Teste de Esforço; Fadiga; Pressão Arterial; Reabilitação.

\section{Cardiorrespiratory responses during two submaximal exercise tests in participants of a cardiac rehabilitation program: preliminary results}

\begin{abstract}
Introduction - The submaximal exercise capacity assesses the functional capacity of the individual, which can be measured by the Six Minute Walk Test (6MWT) and by the Four Minute Step Test (4MST). Objective - To compare the cardiorrespiratory responses and perceived exertion between the 6MWT and 4MST in patients undergoing coronary artery bypass grafting (CABG) participants in a cardiac rehabilitation program. Methodology - Cross-sectional study composed of seven men (58.7 \pm 9.37 years). The cardiovascular responses were analyzed (peripheral oxygen saturation - SpO2; heart rate - HR; systolic blood pressure - SBP; diastolic blood pressure - DBP), dyspnea and lower limb (LL) fatigue were assessed by the modified Borg scale. The tests were administered in according to American Thoracic Society (ATS, 2002) guidelines. Results - Significant differences were observed $(p<0,05)$ in the deltas of SBP, DBP between the tests. Conclusion - Our results suggest that both the 6MWT as the 4MST estimate submaximal exercise capacity, but the 4MST requires a higher metabolic demand, observed by greater increase in blood pressure.
\end{abstract}

Descriptors: Cardiovascular Diseases; Exercise Test; Fatigue; Arterial Pressure; Rehabilitation.

${ }^{1}$ Graduanda em Fisioterapia na Universidade Federal de Santa Maria (UFSM), Santa Maria, RS, Brasil.

${ }^{2}$ Doutura em Ciências da Saúde pela Universidade do Extremo Sul Catarinense (UNESC), Criciúma, SC, Brasil.

${ }^{3}$ Doutorando em Ciências da saúde no Instituto de cardiologia do Rio Grande do Sul (IC/FUC), Porto Alegre, RS, Brasil.

${ }^{4}$ Doutor em Medicina pela Universidade de São Paulo (USP), São Paulo, SP, Brasil.

${ }^{5}$ Mestre em Ciências médicas pela Universidade Federal do Rio Grande do Sul (UFRGS), Porto Alegre, RS, Brasil.

${ }^{6}$ Doutora em Ciências da Saúde pela Universidade Federal do Rio Grande do Sul (UFRGS), Porto Alegre, RS, Brasil.

${ }^{7}$ Doutora em Ciências médicas pela Universidade Federal do Rio Grande do Sul (UFRGS), Porto Alegre, RS, Brasil. 


\section{Introdução}

Os testes físicos funcionais são considerados formas alternativas de avaliação da tolerância ao exercício, sendo de fácil realização, não requerem equipamentos de alto custo, e são de simples aplicação na prática clínica'-2. Dentre esses testes destacam-se o teste do degrau de quatro minutos (TD4) e o teste de caminhada de seis minutos (TC6m), entretanto são escassos os estudos que comparam as respostas cardiovasculares e respiratórias em pacientes submetidos à cirurgia de revascularização do miocárdio (CRM)3.

O TC6m é utilizado para avaliar a resposta do indivíduo ao exercício e propicia uma análise global dos sistemas respiratório, cardíaco e metabólico ${ }^{4}$. Apresenta algumas vantagens como sua simplicidade e exigências tecnológicas mínimas, além disso, é um teste já padronizado e permite que os sinais vitais e sintomas sejam mensurados durante sua execução ${ }^{5-6}$. Neste teste o indivíduo é avaliado perante a distância percorrida em uma superfície plana durante seis minutos. Além deste desfecho, pode-se ainda avaliar a fadiga muscular e a sensação de dispneia, através da Escala de Borg modificada e aferição da saturação periférica de oxigênio ${ }^{7}$.

Já o TD4 normalmente é realizado com cardiopatas ou portadores de doenças respiratórias no qual o indivíduo apenas sobe e desce um degrau, avaliando as respostas fisiológicas ${ }^{8}$ e indiretamente equilíbrio, coordenação, força muscular, trabalho aeróbico e saturação de oxigênio no organismo $0^{9-10}$. Tal teste tem se mostrado útil na avaliação da tolerância ao exercício ${ }^{11}$, visto que impõe alta demanda metabólica quando comparado ao TC6m, além disso, é uma modalidade de baixo custo para predizer a aptidão cardiorrespiratória, já que, requer mínimo espaço físico, pouco ou nenhum equipamento, exigindo pouca prática para sua realização e normalmente tendo curta duração ${ }^{12}$.

Recente metanálise demonstrou que grande parte do sucesso dos programas de reabilitação cardíaca (RC) é devido à terapia baseada no exercício físico, sendo esta considerada a estratégia central destes programas ${ }^{13}$. 0 papel do fisioterapeuta no programa de RC tem sua ênfase na recuperação dos aspectos físicos, cabendo a ele também 0 treinamento e a investigação do potencial para o trabalho, bem como o teste de trabalho simulado e 0 teste ao exercício ${ }^{14}$. Assim, os testes submáximos como o TC6m e o TD4 permitem uma avaliação do estado funcional do sistema cardiovascular e/ou respiratório na saúde e doença, além da avaliação dos programas de RC ${ }^{15}$.

Devido a importância clínica dos dois testes, este estudo teve por objetivo comparar as respostas cardiorrespiratórias, sensação de dispneia e fadiga de membros inferiores entre o TD4 e o TC6m em pacientes submetidos à CRM participantes de um programa de RC.

\section{Metodologia}

O presente estudo é de caráter transversal, realizado no período de julho a dezembro de 2012. Aamostra foi selecionada de forma não probabilística e intencional. Foram avaliados sete pacientes do sexo masculino com média de idade de $58,71 \pm 9,37$ anos, que estavam em acompanhamento regular no ambulatório de cardiologia, e eram pertencentes ao Programa de Reabilitação Cardíaca (Fase II) do Serviço de Cardiologia do Hospital Universitário de Santa Maria (HUSM). Foram incluídos os indivíduos que participavam do programa de reabilitação cardíaca por pelo menos seis meses, classe funcional I segundo critérios da New York Heart Association ${ }^{16}$ (obtida pelo teste ergométrico), fração de ejeção do ventrículo esquerdo (FEVE) normal (determinada por ecocardiografia) e função pulmonar normal (obtida por meio de espirometria). Sendo considerados critérios de exclusão a angina instável, tromboflebite atual, embolia recente, infecção sistêmica aguda, bloqueio AV de $3^{\circ}$ grau (sem marcapasso), pericardite ou miocardite aguda, arritmia não controlada, insuficiência ou estenose mitral ou aórtica graves sem tratamento adequado, insuficiência cardíaca descompensada, hipertensão arterial sistêmica (HAS) descontrolada (pressão arterial sistólica - PAS $\geq 200$ e/ou pressão arterial diastólica - PAS > $110 \mathrm{mmHg}$ ), depressão do segmento ST > $2 \mathrm{~mm}$, problemas ortopédicos ou neurológicos graves, diabetes mellitus descontrolada, labirintite, doença sistêmica aguda ou febre de origem desconhecida ou qualquer outra doença metabólica descompensada.

O estudo foi aprovado pelo Comitê de Ética em Pesquisa com Seres Humanos da Instituição sob número 284.026, CAAE,16149813.3.0000.5346. Todos os participantes assinaram o termo de consentimento livre e esclarecido previamente à coleta dos dados.

Os testes foram aplicados sempre por dois avaliadores (previamente treinados), e realizados em dias não consecutivos, 
com intervalo de no mínimo 24 horas, primeiro realizou-se o TC6m e, posteriormente o TD4. Cada teste foi realizado duas vezes com um intervalo mínimo de 30 minutos entre eles, para eliminar 0 efeito do aprendizado ${ }^{5}$, com o melhor desempenho utilizado para análise, sendo todas as avaliações realizadas no período da tarde para evitar diferentes respostas fisiológicas devido a mudanças circadianas. Além disso, todos os pacientes foram orientados inicialmente a não ingerir cafeína, bebidas alcoólicas ou quaisquer outros estimulantes nos dias das coletas de dados e nem realizar atividades extenuantes no dia anterior.

OTC6m foi realizado num corredor plano de 30 metros de comprimento e 1,5 metros de largura, demarcados a cada 3 metros. Antes de iniciar o teste, foram aferidas a PAS, PAD - através de estetoscópio (BD ${ }^{\circledR}$, Brasil) e esfigmomanômetro $\left(\mathrm{BD}^{\circledR}\right.$, Brasil), pelo método auscultatório direto - $\mathrm{FC}, \mathrm{SpO}_{2}$ por meio de um oxímetro de pulso portátil (Modelo $8500 \mathrm{~A}$; Nonin Medical Inc.,Plymouth, MN, EUA) - e a SD e SFMMII por meio da escala de Borg modificada CR-10. O teste consistiu de uma caminhada na qual o paciente foi orientado a percorrer a maior distância possível por um período de seis minutos, com incentivo padronizado a cada minuto ${ }^{5}$. O paciente foi acompanhado pelo fisioterapeuta durante os seis minutos, com monitorização contínua, porém foram registrados os valores de $\mathrm{FC}, \mathrm{SpO}_{2}$ e sensação subjetiva de dispneia no repouso, $2^{\circ}, 4^{\circ}$ e $6^{\circ}$ minutos. Os valores obtidos foram comparados com o predito na literatura ${ }^{7}$.

O TD4 foi realizado em um degrau de $20 \mathrm{~cm}$ de altura, com $80 \mathrm{~cm}$ de comprimento, $40 \mathrm{~cm}$ de largura e piso antiderrapante ${ }^{3}$. Os princípios gerais do TD4 foram também baseados nas recomendações estabelecidas pela American Thoracic Society ${ }^{5}$, e a capacidade funcional foi registrada pelo número total de subidas no degrau com os dois pés. 0 teste teve início com o paciente em pé, sendo esse instruído a subir e descer o degrau no seu próprio ritmo durante 4 minutos e a interromper temporariamente o teste de acordo com sua necessidade, além disso, o teste foi executado sem o apoio dos membros superiores (MMSS), os quais permaneceram estacionários ao longo do corpo. Foram mensurados os mesmos parâmetros fisiológicos do TC6m.

\section{Análise estatística}

Para a análise dos resultados foi utilizado o software SPSS (SPSS for Windows 20.0, Chicago, Illinois, EUA), sendo os dados expressos em mediana e intervalo interquartil. Para comparar as variáveis intra-grupo, como $\mathrm{SpO}_{2}, \mathrm{FC}$, pressão arterial e percepção de esforço, foi utilizado o teste de Wilcoxon. As comparações dos deltas das respostas cardiorrespiratórias entre os grupos frente à realização do TC6m e TD4 e a distância prevista no TC6m e seu valor predito, foram realizadas através do teste $U$ de Mann-Whitney. Foi considerado significativo um $p<0,05$.

\section{Resultados e discussão}

As variáveis demográficas, clínicas, antropométricas, distância percorrida no TC6m e o número de degraus percorridos no TD4 estão demonstradas na Tabela 1. A amostra foi composta por 07 pacientes, sendo todos do gênero masculino, com mediana de idade de 58 (55 - 68) anos, o IMC de 26,6 $(23,7-31,1) \mathrm{Kg} / \mathrm{m}^{2}$, a distância percorrida no TC6m foi de 461,6 (397,7 - 468,4) metros e o número de degraus percorridos no TD4 de 76 (71 - 94) degraus. É importante ressaltar que não houve perda de pacientes após a entrada no estudo.

Tabela 1 - Variáveis demográficas, clínicas, antropométricas, distância percorrida no TC6m e número de degraus percorridos no TD4 da amostra.

\begin{tabular}{c|c|c|c|c|c|c|c}
\hline Sujeito & CF (NYHA) & FEVE (\%) & $\begin{array}{c}\text { Tempo após } \\
\text { CRM (Meses) }\end{array}$ & Idade (anos) & IMC $\left(\mathrm{kg} / \mathrm{m}^{2}\right)$ & TC6m (m) & TD4 (degraus) \\
\hline 1 & I & 69 & 12 & 55 & 31,11 & 460,7 & 96 \\
\hline 2 & 1 & 54 & 13 & 57 & 23,77 & 475,9 & 76 \\
\hline 3 & 1 & 60 & 12 & 68 & 23,75 & 564,0 & 89 \\
\hline 4 & 1 & 68 & 11 & 58 & 29,18 & 390,9 & 75 \\
\hline 5 & 1 & 67 & 12 & 72 & 26,62 & 465,9 & 67 \\
\hline 6 & 1 & 70 & 12 & 58 & 33,08 & 462,6 & 71 \\
\hline 7 & 1 & 58 & 12 & 43 & 25,17 & 440,0 & 94 \\
\hline
\end{tabular}

CF: classe funcional; FEVE: fração de ejeção do ventrículo esquerdo; IMC: índice de massa corporal; NYHA: New York Heart Association; TC6m: distância percorrida no TC6m; TD4: número de degraus subidos. 
Tabela 2 - Comparação das respostas cardiorrespiratórias e percepção de esforço entre o TC6m e TD4

\begin{tabular}{|c|c|c|c|c|c|c|c|c|c|}
\hline \multirow[t]{2}{*}{ Variáveis } & \multicolumn{4}{|c|}{ TC6m (n=7) } & \multicolumn{4}{|c|}{ TD4 (n=7) } & \multirow[t]{2}{*}{$p$-valor* } \\
\hline & Inicial & Final & $\begin{array}{c}\text { Variação } \\
(\Delta)\end{array}$ & $p$-valor ${ }^{\beta}$ & Inicial & Final & $\begin{array}{c}\text { Variação } \\
(\Delta)\end{array}$ & $p$-valor ${ }^{\beta}$ & \\
\hline \multicolumn{10}{|c|}{ Cardiorrespiratórias } \\
\hline SpO2 (\%) & $98(96-98)$ & $93(93-94)$ & $5(3-5)$ & $<0,001$ & $98(97-99)$ & $94(94-95)$ & $4(2-4)$ & $<0,001$ & 0,190 \\
\hline $\mathrm{FC}(\mathrm{bpm})$ & $70(67-75)$ & $99(94-105)$ & $26(25-38)$ & $<0,001$ & $63(56-71)$ & $104(85-107)$ & $33(22-44)$ & 0,001 & 0,445 \\
\hline PAS (mmHg) & $\begin{array}{c}120(110- \\
125)\end{array}$ & $\begin{array}{c}130(130- \\
140)\end{array}$ & $15(10-15)$ & $<0,001$ & $\begin{array}{c}100(100- \\
110)\end{array}$ & $\begin{array}{c}140(125- \\
150)\end{array}$ & $30(15-40)$ & 0,002 & 0,032 \\
\hline $\mathrm{PAD}(\mathrm{mmHg})$ & $75(60-90)$ & $85(75-100)$ & $10(5-15)$ & 0,003 & $60(50-75)$ & $80(75$ - 90) & $15(10-20)$ & 0,005 & 0,048 \\
\hline \multicolumn{10}{|l|}{$\begin{array}{l}\text { Percepção } \\
\text { de esforço }\end{array}$} \\
\hline SD & $0(0-0)$ & $1(1-3)$ & $1(1-2)$ & 0,003 & $0(0-0)$ & $2(1-2)$ & $2(1-2)$ & 0,001 & 0,957 \\
\hline SFMMII & $0(0-0)$ & $4(3-6)$ & $4(3-6)$ & 0,018 & $0(0-0)$ & $4(3-6)$ & $4(3-6)$ & 0,018 & 1,000 \\
\hline
\end{tabular}

Sp02:Saturação periférica de oxigênio; FC: Frequência cardíaca; PAS: Pressão arterial sistólica; PAD: Pressão arterial diastólica; SD: sensação de dispneia; SFMMII: sensação de fadiga nos membros inferiores; $\Delta$ : Variação; MMII: membros inferiores. Valores descritos em mediana e intervalo interquartil. $\beta$ Comparação entre os valores iniciais e finais. ${ }^{*}$ Comparação entre o delta $(\Delta)$ de variação do TC6m e TD4. Nível de significância para $p<0,05$

O presente estudo, até onde sabemos, foi o primeiro a avaliar as respostas cardiorrespiratórias e percepção de esforço entre o TC6m e o TD4 em pacientes submetidos à CRM participantes de um programa de RC. Nossos resultados demonstraram que houve variação significativa nas respostas cardiorrespiratórias e na percepção do esforço com a realização dos dois testes, entretanto, ao compararmos estas variações entre os grupos através do delta, constatou-se que esta foi diferente apenas quanto à PAS $(p=0,032)$ e PAD $(p=0,048)$. Tal achado pode estar associado ao fato de que, mesmo sendo o TD4 um teste submáximo, o trabalho contra a gravidade e o uso de grupamentos musculares não utilizados com frequência nas atividades de vida diária torna, durante o teste, as demandas metabólicas e ventilatórias mais intensas para o mesmo, podendo acarretar respostas fisiológicas diferentes do TC6m ${ }^{17-18}$. Outra hipótese plausível para esse resultado é a de que, no teste do degrau, há uma maior participação da musculatura das articulações dos membros inferiores, percebendo-se o envolvimento de diferentes ângulos capazes de provocar alterações na resposta pressórica. Além disso, vale ressaltar que diferentemente do TC6m, em que há apenas um componente horizontal no trabalho executado, no teste do degrau há o acréscimo do componente de deslocamento vertical, o que tende a aumentar o nível de exigência do mesmo ${ }^{19}$.

São inexistentes os estudos que tenham analisado as diferentes respostas cardiorrespiratórias e percepção de esforço obtidas entre o TC6m e o TD4 dentro da prática clínica da reabilitação cardíaca. Entretanto, é importante mencionar o estudo de Silva et al. ${ }^{20}$ que, apesar de ter sido conduzido com uma população (pacientes em reabilitação por acidente vascular encefálico) diferente da do nosso estudo, comparou o comportamento das variáveis cardiorrespiratórias durante o TC6m e o teste do degrau de seis minutos (TD6), não encontrando diferença significativa entre as variáveis fisiológicas analisadas ( $\mathrm{SpO}_{2}$, FC, PAS, PAD e percepção de esforço), nem associação entre a distância percorrida no TC6m e o número de degraus no TD6. Schnaider e Karsten21 avaliaram a tolerância ao exercício em oito pacientes do gênero masculino, hospitalizados devido à exacerbação da DPOC, através do TC6m e do TD6. Os autores constaram resultados semelhantes aos encontrados no presente estudo, pois ambos os testes produziram efeitos equivalente quanto às variáveis cardiorrespiratórias ( $\mathrm{FC}, \mathrm{SpO}_{2}$ e frequência respiratória) e de percepção de esforço.

Em estudo conduzido por Pasqualoto ${ }^{22}$, no qual objetivou comparar as respostas fisiológicas obtidas no teste de exercício cardiopulmonar com três testes de exercício submáximo (incluindo o TD6 e o TC6m) em pacientes com doença pulmonar obstrutiva crônica (DPOC), os resultados mostraram que as respostas fisiológicas foram semelhantes entre ambos.

Em relação à sensação de dispneia, não houve diferença significante entre os testes, demonstrando demandas perceptuais similares. Puente-Maestu et al. ${ }^{23}$, em estudo também com portadores de DPOC, encontraram resultados que vão ao encontro dos obtidos no presente estudo, pois observaram que a SD não se diferiu de forma significante entre testes submáximos em cicloergômetro nas quatro intensidades de trabalho avaliadas.

Estudo prévio realizado entre adolescentes asmáticos e saudáveis visando comparar o desempenho físico e as respostas obtidas no TC6m e no TD6 obteve, como um dos resultados, menores valores de FC e maiores valores de 
SFMMII no grupo asmático durante o TD6. Os autores deste estudo consideram que a resposta mais elevada da FC e da SFMMII, deve-se ao fato deste teste provocar maior trabalho em grupamentos musculares contra a gravidade, não utilizados frequentemente na prática de atividade de vida diária, tornando as demandas metabólicas e ventilatórias mais intensas que o $\mathrm{TC}^{2} \mathrm{~m}^{24}$.

Travensolo et al. 25 compararam em 38 indivíduos idosos (68,5 $\pm 2,1$ anos) o delta de variação das variáveis FC, PAS e PAD entre o TD6 e o TC6m, onde os indivíduos que realizaram o TD6 apresentaram incremento significativamente maior $(p=0,011)$ somente na variação da FC, não havendo diferença significativa entre os deltas da PAS e PAD. Tais achados se contrapõem aos do presente estudo, pois houve diferença significativa nos deltas de variação da PAS e PAD, entretanto não constatamos diferença no delta da FC entre o TC6m e o TD4.

Cardoso et al.26 compararam o TD5 com o TC6m em relação à SpO2 (>5\%) durante exercícios em portadores de DPOC estável e normóxicos, concluindo que o teste de degrau é efetivo na deteç̧ão da dessaturação. Em um estudo longitudinal, Stephan et al.27 correlacionaram a dessaturação de oxigênio durante o TD4 com a sobrevida em pacientes com fibrose pulmonar idiopática. Constatou-se que SpO2 < 89\% foi um forte preditor de mortalidade nessa população. No entanto, é importante ressaltar que no nosso estudo, o teste foi realizado em apenas 4 minutos, dessa forma infere-se que este tempo possa ter sido insuficiente para provocar alterações significativas nesta variável, já que se tratavam de indivíduos com função pulmonar normal.

É importante mencionar que no presente estudo, os pacientes apresentaram distância percorrida no TC6m significativamente menor que os valores preditos de normalidade, segundo a equação de referência de Enright e Sherrill7, conforme demonstrado na Figura 01.

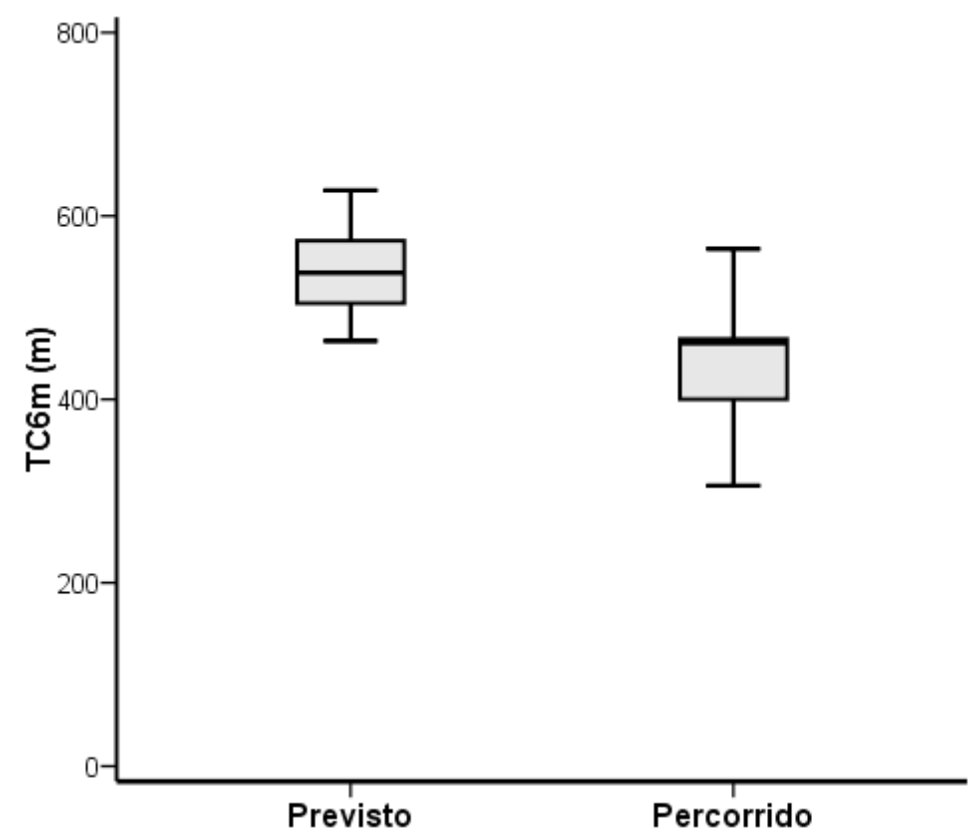

Figura 1. Comparação entre a distância predita e a percorrida no Teste de Caminhada de 6 minutos (TC6m). Valores expressos em mediana e intervalo interquartil.

Esses dados sugerem que pacientes pós-CRM, submetidos a um programa de reabilitação cardíaca, podem apresentar redução da capacidade funcional em relação ao esperado para indivíduos saudáveis. No entanto, no presente estudo, a distância percorrida no TC6min (461,6 m) foi semelhante a observada em estudo prévio (439 $\pm 171 \mathrm{~m}$ ) envolvendo 52 pacientes que mantiveram-se ativos após a $\mathrm{CRM}^{28}$.

É considerada como uma limitação do estudo o reduzido tamanho amostral, entretanto esse aspecto não invalida os resultados preliminares obtidos até o presente momento, principalmente em função de que a comparação entre TC6min e TD4 é inédita para a população avaliada. 


\section{Conclusão}

Nossos resultados demonstraram que houve variação significativa nas respostas cardiorrespiratórias e na percepção do esforço nos dois testes realizados, entretanto, em função do achado do maior incremento da pressão arterial, sugerese que o TD4 exige uma maior demanda metabólica quando comparado ao TC6M. Além disso, nosso estudo sugere que o teste do degrau pode ser uma alternativa para substituir o teste de caminhada quando não há um espaço físico amplo para sua realização, porém fazem-se necessários ainda futuros estudos para a sua padronização.

\section{Referências bibliográficas}

1. Casas A, Vilaro J, Rabinovich R, Mayer A, Barberà JA, Rodriguez-Roisin R, et al. Encouraged 6-min walking test indicates maximum sustainable exercise in COPD patients. Chest. 2005;128(1):55-61.

2. Cataneo DC, Cataneo AJM. Acurácia do teste de escada utilizando o consumo máximo de oxigênio como padrão-ouro. J Bras Pneumol. 2007;33(2):128-33.

3. Dal Corso S, Duarte SR, Neder JA, Malaguti C, Fuccio MB, Castro Pereira CA, et al. A step test to assess exerciserelated oxygen desaturation in interstitial lung disease. Eur Respir J. 2007;29(2):330-6.

4. Li AM, Yin J, Yu CC, Tsang T, So HK, Wong E, et al. The six-minute walk test in healthy children: reliability and validity. Eur Respir J. 2005;25(6):1057-60.

5. ATS Committee on Proficiency Standards for Clinical Pulmonary Function Laboratories. ATS statement: guidelines for the six-minute walk test. Am J Respir Crit Care Med. 2002;166(1):111-7.

6. Asociación Argentina de Medicina Respiratoria [homepage on the Internet]. Buenos Aires: Asociación Argentina de Medicina Respiratoria. [cited 2010 Jun 1]. López JO, Carbone LS. Prueba de marcha de seis minutos. Available from: http://www.aamr.org.ar/cms/archivos/secciones/fisiopatologia/prueba6minut.doc

7. Enright PL, Sherrill DL. Reference equations for the six-minute walk in healthy adults. Am J Respir Crit Care Med 1998;158(5 Pt 1): 1384-1387.

8. Andrade CHS, Cianci RG, Malaguti C, Dal Corso C. O uso de testes do degrau para a avaliação da capacidade de exercício em pacientes com doenças pulmonares crônicas. J. Bras. Pneumol. 2012; 38 (1).

9. Campos, MV. Teste do Degrau: avaliação e treinamento Disponível em:<http://www.revistavigor.com.br/2009/03/26/ teste-do-degrau-avaliacao-e-treinamento/>. Acesso em: 05/07/2013.

10. Pessoa BV, Jamami M, Basso RP, Regueiro EMG, Di Lorenzo VAP, Costa D. Teste do degrau e teste da cadeira: comportamento das respostas metábolo-ventilatórias e cardiovasculares na DPOC. Fisioter Mov. 2012;25(1):105-15.

11. Temas em revisão. Sociedade Brasileira de Pneumologia e Tisiologia. Disponível em:< http://www. sbpt.org.br $>$. Acesso em: 06/07/2013.

12. American College of Sports Medicine. Diretrizes do ACSM para os testes de esforço e sua prescrição. 7. ed. Rio de Janeiro: Guanabara Koogan; 2007. p. 216-7.

13. Oldridge $\mathrm{N}$. Exercise-based cardiac rehabilitation in patients with coronary heart disease: meta-analysis outcomes revisited. Future Cardiol. 2012;8(5):729-51

14. Pryor, J.A; Webber, B.A. Fisioterapia para problemas Respiratórios e Cardíacos. 2 ed. Rio de Janeiro: Guanabara Koogan; 2002.

15. Busch JC, Lillou D, Wittig G, Bartsch P, Willemsen D, Oldridge N, Bjarnason-Wehrens B. Resistance and balance training improves functional capacity in very old participants attending cardiac rehabilitation after coronary bypass surgery. J Am Geriatr Soc. 2012;60(12):2270-6.

16. New York Heart Association (NYHA). Nomenclature and Criteria for Diagnosis of Diseases of the Heart and Great Vessels. 9th ed. Boston, Mass: Little, Brown \& Co; 1994:253-256.

17. Dal Corso S, Duarte SR, Neder JA, Malaguti C, de Fuccio MB, de Castro Pereira CA, Nery LE. A step test to assess exercise-related oxygen desaturation in interstitial lung disease. Eur Respir J. 2007;29(2):330-6.

18. Swinburn CR, Cooper BG, Mould H, Corris PA, Gibson GJ. Adverse effect of additional weight on exercise against gravity in patients with chronic obstructive airways disease. Thorax. 1989;44(9):716-20.

19. Marrara KT, Marino DM, Jamami M, Junior DO, Lorenzo VAPD. Responsividade do teste do degrau de seis minutos a um programa de treinamento físico em pacientes com DPOC. J Bras Pneumol. 2012;38(5):579-587. 
20. Silva, TD, et al. Comparison between the six-minute walk test and the six-minute step test in post stroke patients. Int Arch Med. 2013: 7;6(1):31.

21. Schnaider J, Karsten M. Testes de tolerância ao exercício em programa de fisioterapia hospitalar após exacerbação da doença pulmonar obstrutiva crônica. Fisioterapia em movimento. 2006:19 (4):119-26.

22. Pasqualoto, AS. Comparação das respostas fisiológicas no teste de exercício cardiopulmonar e em três testes de exercício submáximo em pacientes com doença pulmonar obstrutiva [tese]. Porto Alegre: Universidade Federal do Rio Grande do Sul; 2009.

23. Puente-Maestu L, Pedro JG, Martínez-Abad Y, Oña JMR, Llorente D, Cubillo JM. Dyspnea, ventilator pattern, and changes in dynamic hyperinflation related to the intensity of constant work rate exercise in COPD. Chest. 2005;128(2):651-6. 24. Basso RP, Jamami M, Pessoa BV, Labadessa IG, Regueiro EMG, Lorenzo VAP. Avaliação da capacidade de exercício em adolescentes asmáticos e saudáveis. Rev Bras Fisioter. 2010; 14 (3):252-258

25.Travensolo CF, Goessler KF, Polito MD. Correlação entre o teste de caminhada de seis minutos e o teste do degrau em idosos. Rev. Bras. Geriatr. Gerontol. Rio de Janeiro, 2013; 16(2):375-383.

26. Cardoso F, Machado MCLO, Leme MD, Tachibana KM, Togeiro S, Jardim JR. Comparação do teste do degrau versus a caminhada de seis minutos em relação à dessaturação de oxigênio durante exercícios em portadores de DPOC estável e normóxicos. Revista Brasileira de Fisioterapia 2000;4(3):25.

27. Stephan S, de Castro Pereira CA, Coletta EM, Ferreira RG, Otta JS, Nery LE. Oxygen desaturation during a 4-minute step test: predicting survival in idiopathic pulmonary fibrosis. Sarcoidosis Vasc Diffuse Lung Dis. 2007;24(1):70-6.

28. Nery RM, Martini MR, Vidor CR, Mahmud MI, Zanini M, Loureiro A, et al. Alterações na capacidade funcional de pacientes após dois anos da cirurgia de revascularização do miocárdio. Rev Bras Cir Cardiovasc. 2010;25(2):224-8.

\section{Marília Severo Vicente}

Endereço para correspondência - Venâncio Aires, 1536, apto 12. Bairro: Centro, CEP 97010-002, Santa Maria, RS, Brasil.

E-mail: mariliasvicente@gmail.com

Currículo Lattes: http://lattes.cnpq.br/2786696166052433

Tamires Daros dos Santos - tamydaros@bol.com.br

Viviane Acunha Barbosa - vivianeab51@yahoo.com.br

Tiago José Nardi Gomes - tiagonardi@yahoo.com.br

Sérgio Nunes Pereira - sergionunespereira@gmail.com

Dannuey Machado Cardoso - dannuey@yahoo.com.br

Carine Cristina Callegaro - ccallegaro84@gmail.com

Isabella Martins de Albuquerque - albuisa@gmail.com

Recebido em 09 de janeiro de 2013.

Publicado em 15 de maio de 2014. 
